\title{
THE INTERSECTION OF THE FREE MAXIMAL IDEALS IN A COMPLETE SPACE
}

\author{
STEWART M. ROBINSON
}

Introduction. In [1, p. 123] Gillman and Jerison prove that if $X$ is realcompact, the intersection of the free maximal ideals in $C(X)$ is $C_{K}(X)$, the ring of functions with compact support. Other authors [2], [3] have proved this result for discrete spaces and $p$-spaces, respectively. This note provides a proof of the fact that the result holds for any space admitting a complete uniform structure. We point out also that unlike that of the Gillman-Jerison theorem, our proof requires no prior construction of $\beta X$, the Stone-Cech compactification of $X$. Since a realcompact space is complete in the structure generated by $C(X)$, our result extends the Gillman-Jerison theorem. (Whether every complete space is realcompact is an unsettled question: its resolution would require a proof of the existence or nonexistence of measurable cardinals.)

We will employ the same terminology as in [1] and assume all spaces are completely regular and all uniform structures Hausdorff. Also, we take our uniform structures to be defined by pseudometrics. For a topological space $X, C(X)$ denotes its ring of real valued continuous functions, and for $f \in C(X), Z_{f}=\{x: f(x)=0\}$. Thus, the support of $f$ is $\mathrm{cl}_{X}\left[X-Z_{f}\right]$ and $C_{K}(X)$ is the ring of functions with compact support.

An ideal $I \subseteq C(X)$ is free provided $\cap\left\{Z_{f}: f \in I\right\}=\varnothing$ and a maximal ideal which is free is called a free maximal ideal. A subset $S \subseteq X$ is $C$-embedded in $X$ provided each $f \in C(S)$ admits a continuous extension to all of $X$.

Lemma. If $f \in C(X)$ and if $X-Z_{f}$ contains a closed, noncompact $C$-embedded subset, then $f$ fails to belong to some free maximal ideal of $C(X)$.

Proof. Let $A \subseteq X-Z_{f}$ satisfy the hypotheses of the lemma. Then, there exists an $h \in C(X)$ such that $A \subseteq Z_{h}$ and $h\left[Z_{f}\right]=1$. Upon noting that $h$ and $f$ cannot belong to the same ideal $\left(f^{2}+h^{2}\right.$ is a unit), we may complete the proof by showing that $h$ belongs to some free maximal ideal $M$. Since $A$ is a closed, noncompact subset of $Z_{h}, Z_{h}$ is not compact; hence, $h$ belongs to some free ideal $I$. According

Presented to the Society, December 11, 1964; received by the editors March 1, 1965. 
to Zorn's lemma, $I$ is contained in a maximal ideal $M$ and since $\cap\left\{Z_{f}: f \in M\right\} \subseteq \cap\left\{Z_{o}: g \in I\right\}=\varnothing, M$ is free.

Theorem. If $X$ admits a complete uniform structure $D$, then the intersection of the free maximal ideals in $C(X)$ is $C_{K}(X)$.

Proof. $C_{K}(X)$ is contained within every free ideal, hence in the intersection of all free maximal ideals.

Conversely, suppose $\operatorname{cl}_{X}\left[X-Z_{f}\right]$ is not compact. As a closed subset of the complete space $(X, D)$, it is complete in the relative structure generated by $D$; in fact, it is the completion of $X-Z_{f}$ in the relative structure. Since $X-Z_{f}$ has a noncompact completion, we infer that for some $d \in D$ and $\rho>0$, there is an infinite closed set $A \subseteq X-Z_{f}$ such that for distinct points $a$ and $a^{\prime}$ in $A d\left(a, a^{\prime}\right) \geqq \rho$. For $g \in C(A)$, the function $G$ defined by:

$$
\begin{aligned}
& G(x)=g(a)(1-3 d(x, a) / \rho) \quad \text { if for some } a \in A, \quad d(x, a) \leqq \rho / 3 \\
& G(x)=0 \quad \text { for other } x
\end{aligned}
$$

is a continuous extension of $g$ to all of $X$. Thus, $A$ is $C$-embedded in $X$ and the conclusion follows from the preceding lemma.

This hypothesis that $X$ is complete is not a necessary condition. The space $W$ of all ordinals less than the first uncountable ordinal is pseudocompact, but not compact. Hence it admits no complete uniform structure. Nevertheless, the one free maximal ideal in $C(W)$ is the set $\{f \in C(W): f$ vanishes on some tail of $W\}$, and this set is precisely $C_{K}(W)$. (This example is constructed in [1] to show that realcompactness is not a necessary condition.)

\section{REFERENCES}

1. L. Gillman and M. Jerison, Rings of continuous functions, Princeton Univ. Press, Princeton, N. J., 1960.

2. I. Kaplansky, Topological rings, Amer. J. Math. 69 (1947), 153-183.

3. C. W. Kohls, Ideals in rings of continuous functions, Fund. Math. 45 (1957), 28-50.

Union College, Schenectady 